\title{
Catasetum (ORCHIDACEAE: CATASETINAE) EN COLOMBIA: LISTA ACTUALIZADA
}

Fecha de recepción: 17 de septiembre de 2015 • Fecha de Evaluación: 25 de febrero de 2016 •

Fecha de aceptación: 18 de marzo de 2016

\section{Catasetum (Orchidaceae: Catasetinae) FOR COLOMBIA: UPDATED LIST}

Miguel M. Bonilla1,2,3, A. Carolina Aguirre ${ }^{1,2}$, Diego Yepes R. ${ }^{1}$, Enmanuel Gallego D. ${ }^{1}$, J. Tupac Otero'.

\section{RESUMEN}

Colombia se considera uno de los países más ricos en orquídeas, a pesar de que ha sido poco estudiado en algunas bioregiones del país. Los registros del género Catasetum corresponden entre 29 a 31 especies, sin embargo, existe un sinnúmero de equivocaciones en la identidad taxonómica interespecífica, como en el uso de sus sinónimos. Por tal motivo, el objetivo del presente trabajo es conocer la diversidad de Catasetum en Colombia. De esta manera, se realizaron salidas de campo, revisión de colecciones, literatura y herbarios. En la lista actualizada se registran 38 especies, que incluyen poco conocidas y confundidas como C. dejeaniorum, C. aff. juruenense, C. mooeri, C. tenebrosum y los híbridos naturales como C. aff. $\times$ apolloi, C. $\times$ dunstervillei y $C$. $\times$ violascens. De las cinco especies endémicas, C. blepharochilum y C. tricorne, se conocen solo de espécimen tipo, y C. lucis es exclusivo de Santander y cultivado, para C. rectangulare y C. villegasii, se realizan comentarios taxonómicos. La Amazonía y los Andes concentran la mayor diversidad y, el Caribe es la zona menos explorada. Además se incluyen los caracteres diagnóstico, rangos altitudinales, relaciones geográficas y los sinónimos utilizados en el país para algunas especies con identidad taxonómica dudosa. También, se resalta la necesidad de realizar exploraciones en zonas de vacíos de colecta, como el Atlántico y, el Amazonas, centro de diversidad del grupo, con la finalidad de establecer futuros planes de manejo y conservación.

Palabras claves: diversidad, híbrido natural, orquídeas, taxonomía.

1 Grupo de Investigación en Orquídeas, Ecología y Sistemática Vegetal, Universidad Nacional de Colombia Sede Palmira.

2 Fundación Diversidad Biológica, Pasto, Nariño.

3 Autor corresponsal: E-mail: mmbonillam@unal.edu.co 


\begin{abstract}
Colombia is considered one of the richest countries in orchids, although it has been little studied in different bioregions of the country. The records of the genus Catasetum are between 29-31 species, however, there are countless mistakes in interspecific taxonomic identity and the use of synonyms. Therefore, the objective of this study was to determine the diversity of Catasetum in Colombia. Thus, field trips, and revision of collections and literature was conducted. An updated list of 38 species is presented, including species little known species such as $C$. dejeaniorum, $C$. aff juruenense, $C$. mooeri, $C$. tenebrosum and natural hybrids such as $C$. aff. $x$ apolloi, C. $\times$ dunstervillei and C. $\times$ violascens. Of the five endemic species, C. blepharochilum and C. tricorne, known only from type specimen, and C. lucis is unique to Santander and cultivated and for C. rectangulare and C. villegasii, taxonomic comments are made. The Amazon and the Andes concentrate most diversity, the Atlantic is the least explored area. Further diagnostic characters, altitudinal ranges, geographical relationships and synonyms used in the country for some species with uncertain taxonomic identity are included. Also, the need for exploration in areas without collections as the Atlantic and the Amazon, center of diversity of the group, in order to establish future management and conservation is highlighted.
\end{abstract}

Key words: diversity, natural hybrid, orchids, taxonomy.

\section{INTRODUCCIÓN}

La familia Orchidaceae Juss. es uno de los grupos de angiospermas con mayor diversidad y abundancia de especies en el planeta, tanto que su cifra se aproxima a 28.000 (Giraldo y Betancur, 2011; Zots, 2013), sin incluir los híbridos artificiales que duplican este estimativo (Bonilla et al. 2014a; Chase et al. 2015). Para Colombia son aproximadamente 4.010 especies concentradas en 232 géneros (Sarmiento, 2007). De las especies registradas para Colombia 1.544 son endémicas, distribuidas preferencialmente en la bioregión andina donde ocurren el $87.2 \%$ de orquídeas del país (Sarmiento, 2007).

Catasetum Rich. ex Kunth hace parte de la subfamilia Epidendroideae Kosteletzky, la tribu Cymbidieae Pfizer y la subtribu Catasetinae Lindl. Esta última posee siete géneros además, de Catasetum: Clowesia Lindl., Cyanaeorchis Barb. Rodr., Cycnoches Lindl., Dressleria Dodson, Galeandra Lindl., Grobya Lindl. y Mormodes Lindl., sensu Chase et al.
(2015). Catasetinae tiene ca. 354 especies, distribuidas en el Neotrópico, a una altura entre 50 -2.000 msnm (Bonilla et al. 2013a; Whitten et al. 2014; Freudenstein y Chase, 2015). Por su parte, Colombia posee seis géneros de Catasetinae (excepto Cyanaeorchis y Grobya), siendo Catasetum el mejor representado en número de especies (29-31 spp.), abundancia y distribución en el país, particularmente en las regiones Andina, Amazonía y Orinoquía (Ortiz y Uribe, 2007; 2014).

Catasetum se caracteriza por presentar pseudobulbos fusiformes y en algunos casos elipsoides, ovados y subcónicos, hojas plicadas que al caerse dejan una especie de espina, inflorescencia axial y flores unisexuales (Holst, 1999). Este taxón presenta dimorfismo sexual, no obstante, en algunas ocasiones aparecen flores intermedias no funcionales (Romero, 2012). Las flores masculinas se utilizan para establecer las diferencias interespecíficas, a través 
de las cerdas (antenas) y el labelo, principalmente, puesto que las flores femeninas, por lo general, presentan labelo en forma de yelmo (Romero, 2012; Gerlach, 2013). Sin embargo, algunas flores masculinas presentan homología floral con las femeninas, un ejemplo es Catasetum discolor Lindl., Catasetum longifolium Lindl. y su híbrido natural, Catasetum $\times$ roseoalbum Lindl, pues las flores masculinas y femeninas son similares (Romero, 2009; Bonilla et al. 2013a).

Catasetum incluye ca. 140 especies en el Neotrópico (Romero-González, 2012), sin embargo, Chase et al. (2015) sugieren que el género alcanza 176 especies. En Colombia, Ortiz y Uribe (2007; 2014) reconocen 29-31 especies, definidas por registros fotográficos, y que relacionan algunos especímenes de herbario en Ortiz (2015) para Catasetum. El objetivo del presente trabajo fue establecer la relación entre especies registradas en fotografías, colecciones botánicas y sinónimos con el fin de definir la lista actualizada de las especies de Catasetum, con comentarios sobre especies dudosas teniendo en cuenta sus localidades, bioregiones y rango altitudinal de procedencia, de tal manera, este conocimiento permitirá a futuro establecer las relaciones morfológicas, dinámica de poblaciones y estado de conservación para las especies.

\section{MATERIALES Y MÉTODOS}

\section{Revisión de herbarios}

Se realizaron visitas y revisión de colecciones botánicas de los herbarios nacionales $\mathrm{COL}, \mathrm{COAH}$, VALLE, CUVC, HUA, HUQ, JAUM, JBB, LLANOS, MEDEL, TULV, TOLI, CDMB, UIS y UDBC. Además se consultaron herbarios internacionales en línea como MO y K y sitios web como The Plant List (http://www. theplantlist.org/) y GBIF (http://www.gbif.org/) para la obtención de información relacionada con registros o especímenes del grupo de estudio.
Para la identificación taxonómica de las especies se realizó la comparación con material de herbario, especímenes tipo y las descripciones de la publicación tipo para cada especie registradas, siguiendo lineamentos de MO y K sensu Chase et al. (2005) y complementado con Romero y Jenny (1993).

\section{Literatura}

Se revisó la literatura botánica nacional e internacional que incluyen reportes de orquídeas. Se obtuvo información de la literatura científica publicada sobre las especies del género Catasetum en Colombia como Camargo y Delgado (2005), Misas (2005), Ortiz y Uribe (2007; 2014), Mejía (2009), Pérez (2009), Romero (2009), Reina et al. (2010), López (2011), Jiménez (2011), Giraldo y Betancur (2012), Bonilla et al. (2013 a,b; 2014a), Martínez et al. (2015) y Ortiz (2015).

\section{Colecciones de campo}

El esfuerzo de colecta de información se realizó en los departamentos de Amazonas, Arauca, Antioquía, Bolívar, Caldas, Casanare, Cauca, Cundinamarca, Huila, Meta, Nariño, Putumayo, Quindío, Risaralda, Santander, Tolima y Valle del Cauca, visitando un total de 100 municipios, que comprenden corredores y senderos ecológicos como bosques y bordes de camino, durante 1 a 10 días por salida, en zonas asociadas a bosque seco. De tal manera, se procedió a colectar muestras para especímenes de herbario, flores en alcohol para antoteca, registro fotográfico de las partes vegetativas y florales e información de pasaporte para cada una de las especies encontradas que corresponde a departamento, municipio, localidad, longitud, latitud y elevación para conocer componentes de diversidad, distribución y taxonomía.

Además, se obtuvo material vegetal vivo y en alcohol, fotografías y observaciones de plantas a través de las Sociedades orquideológicas, exposiciones 
nacionales, cultivadores y colecciones privadas durante el periodo de estudio.

\section{Inventario y distribución}

Con la información obtenida se estableció una lista con el taxón, su autor y fecha de publicación, los caracteres infrafamilia (subfamilia, tribu, sustribu) hasta género, seguido se hizo relación con lo reportado en otros países como departamentos en Colombia utilizando siglas internacionales, de igual manera se estableció el área geográfica con el rango altitudinal de cada una de las especies.

Sumado a lo anterior, con los sitios de colecta se constituyó una base de datos en el programa de Excel, paquete Office, 2010 con las coordenadas geográficas. De acuerdo con Bonilla et al. (2014b), los datos fueron analizados con el programa DIVA-GISC versión 7.5 para establecer la distribución mediante puntos en el mapa de Colombia, (con capa de elevación para Suramérica), donde se registra la presencia del género en cada uno de los departamentos.

\section{Taxonomía}

Se realizaron comentarios taxonómicos sobre las especies que tienen identidad dudosa en el país y registradas en listados se conoce de Catasetum (Ortiz y Uribe, 2007; 2014; Ortiz, 2015). De esta manera, a criterio de las observaciones de campo, herbario y literatura registrada, se complementó la descripción morfológica, distribución por países y en Colombia, y el rango de distribución altitudinal (Schweinfurth, 1960; Schweinfurth, 1970; Romero y Carnevali, 1989a; Romero y Carnevali, 1989b; Carr, 2006; Ortiz y Uribe, 2007; Benelli y Grade, 2008; Carr, 2008; Bonilla et al. 2014a). Para las especies con información escasamente disponible se obtuvieron datos de antoteca, planta viva o espécimen de herbario, con la finalidad de establecer su morfometría e ilustración científica de la flor y la planta.

\section{RESULTADOS Y DISCUSIÓN}

\section{Diversidad florística y taxonomía}

La diversidad de Catasetum registrada para Colombia por Ortiz y Uribe $(2007,2014)$ está entre las 29 y 31 especies. La lista actualizada establece 38 especies para el territorio colombiano (Tabla 1), que incluye un grupo de especies conocidas de fotografías y confundidas con otras, como los híbridos naturales: C. aff. $\times$ apolloi, C. $\times$ dunstervillei y C. $\times$ violascens y especies como C. dejeaniorum, C. aff. Juruenense, C. mooeri, C. tenebrosum poco conocidas o determinadas con el nombre de otras ya registradas para el país. Aunque, Ortiz (2015) establece 34 especies para Colombia, las especies como $C$. caucanum y $C$. fineatum son sinónimos de C. tabulare, y C. integerrinum es confundido con C. maculatum. De tal manera, la diversidad de las especies de Catasetum encontradas en Colombia, define que es el grupo más representativo de la subtribu Catasetinae (Bonilla et al. 2013a). Además, representa el $21 \%$ en relación con las especies que se conocen a nivel mundial, concentradas en el Neotrópico (Chase et al. 2015).

El último inventario de plantas vasculares de Colombia, Ortiz (2015) da a C. caucanum Schltr. y C. fineatium L. Linden \& Cogn. el estatus de especies. Sin embargo, Romero y Jenny (1993) establecen la lista de verificación de especies, variedades e híbridos naturales en Catasetum, donde determinan el lectotipo de $C$. caucanum en el herbario $G$ y, añaden una nota que establece que esta especie es indistinguiblemente una flor intermedia de C. tabulare. Igualmente, C. finetianum L. Linden \& Cogn. que se designa lectotipo en herbario BR es considerado un sinónimo de C. tabulare (Romero y Jenny, 1993). De tal manera, estas dos especies consideradas endémicas para Colombia y conocida solo de especímenes de herbario hacen parte de la variación intraespecífica de C. tabulare (Fig. 1). 


\begin{tabular}{|c|c|c|c|c|c|c|c|c|c|c|c|c|c|c|c|c|c|}
\hline 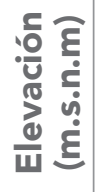 & & $\begin{array}{l}\text { O্ণ } \\
\text { †े } \\
8 \\
8\end{array}$ & $\begin{array}{l}\stackrel{8}{+} \\
\stackrel{1}{\circ}\end{array}$ & $\begin{array}{l}8 \\
\frac{1}{0} \\
\stackrel{1}{n}\end{array}$ & & $\begin{array}{l}8 \\
8 \\
\dot{1} \\
0\end{array}$ & $\begin{array}{l}8 \\
0 \\
\dot{1} \\
8\end{array}$ & 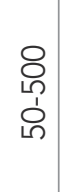 & $\frac{8}{8}$ & 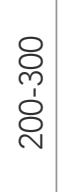 & 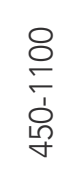 & $\begin{array}{l}8 \\
\text { ధ̊ } \\
\text { ○े }\end{array}$ & 8 & & $\begin{array}{l}8 \\
\stackrel{1}{0} \\
8\end{array}$ & $\begin{array}{l}\stackrel{8}{0} \\
\stackrel{0}{1} \\
\stackrel{1}{\wedge}\end{array}$ & $\begin{array}{l}8 \\
0 \\
0 \\
\text { pे }\end{array}$ \\
\hline 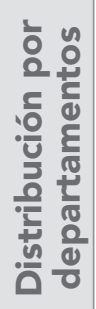 & & $\frac{\frac{\pi}{\sigma}}{\sigma}$ & 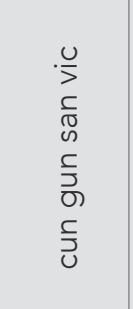 & $\begin{array}{l}\tilde{R} \\
\frac{\tilde{\sigma}}{\sigma}\end{array}$ & శ్ & 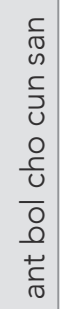 & 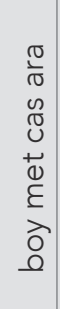 & 亏ั & 亏ั5 & $\begin{array}{l}\frac{u}{0} \\
\frac{0}{0} \\
\frac{c}{w}\end{array}$ & 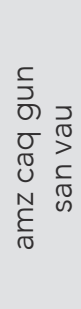 & $\stackrel{\vec{\otimes}}{\varepsilon}$ & $\begin{array}{l}\mathrm{z} \\
\mathrm{a}\end{array}$ & $\begin{array}{l}\frac{u}{O} \\
\frac{0}{d} \\
\frac{c}{w}\end{array}$ & $\stackrel{\vec{\oplus}}{\varepsilon}$ & $\begin{array}{l}\mathbb{r} \\
\mathbb{0} \\
c\end{array}$ & $\begin{array}{l}\tilde{E} \\
\underset{\sigma}{E}\end{array}$ \\
\hline 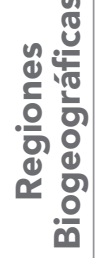 & & 'ำ & 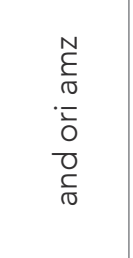 & $\begin{array}{l}N \\
\stackrel{N}{\sigma}\end{array}$ & $\begin{array}{l}\stackrel{\sigma}{\varepsilon} \\
\frac{\varepsilon}{\sigma}\end{array}$ & 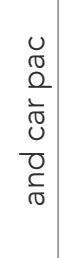 & 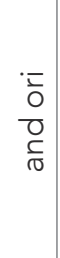 & $\begin{array}{l}\bar{I} \\
0 \\
\bar{O} \\
\frac{C}{\sigma} \\
N \\
\frac{E}{\sigma}\end{array}$ & $\underset{\frac{\varepsilon}{\sigma}}{\mathcal{E}}$ & $\frac{N}{\tilde{E}}$ & $\begin{array}{l}\bar{\Sigma} \\
N \\
\tilde{N} \\
\frac{1}{\pi} \\
\frac{0}{0} \\
\frac{1}{\pi}\end{array}$ & 'ॅ & $\underset{\widetilde{N}}{\tilde{E}}$ & $\begin{array}{l}N \\
\frac{N}{\sigma}\end{array}$ & 'ॅ & $\begin{array}{l}\bar{O} \\
\frac{D}{\sigma}\end{array}$ & $\underset{\sigma}{N}$ \\
\hline 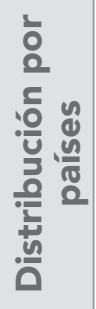 & & $\begin{array}{l}\overline{0} \\
\overline{0} \\
\overline{0}\end{array}$ & 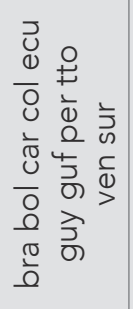 & $\begin{array}{l}\frac{\bar{d}}{\mathrm{~d}} \\
\frac{\mathrm{a}}{\mathrm{O}}\end{array}$ & $\overline{0}$ & 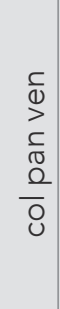 & $\begin{array}{l}\frac{c}{0} \\
0 \\
\lambda \\
0 \\
0 \\
0 \\
0 \\
0 \\
0 \\
0 \\
0\end{array}$ & 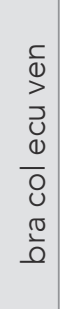 & 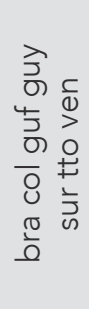 & $\frac{4}{\frac{4}{3}}$ & 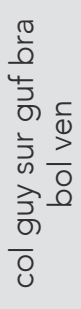 & 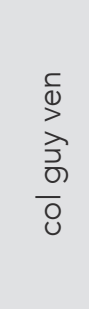 & $\begin{array}{l}\overline{0} \\
\overline{0} \\
\overline{0}\end{array}$ & $\frac{c}{\stackrel{c}{\rho}}$ & 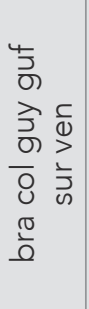 & $\overline{\mathrm{o}}$ & 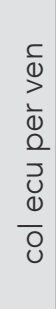 \\
\hline 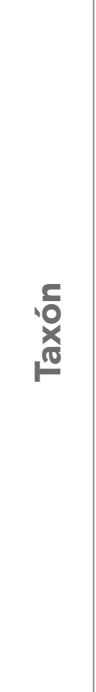 & 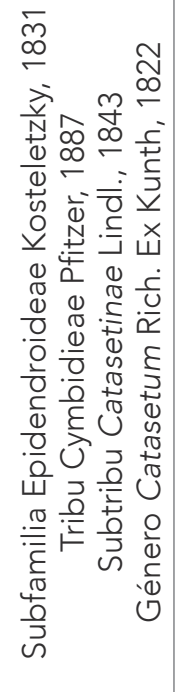 & 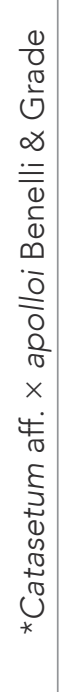 & 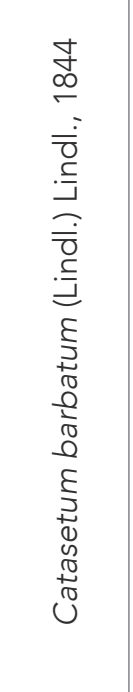 & 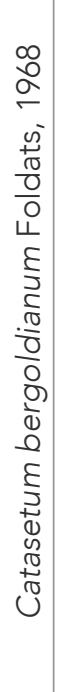 & 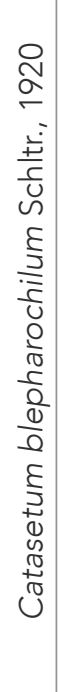 & 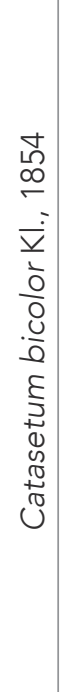 & 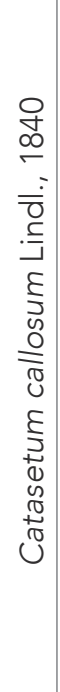 & 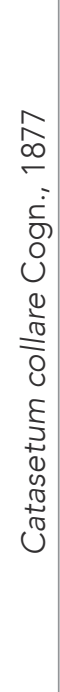 & 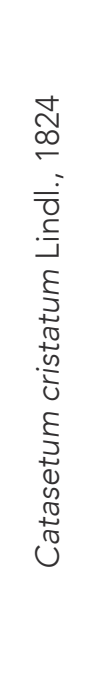 & 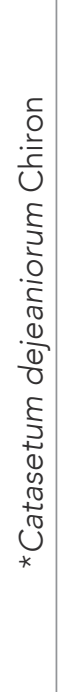 & 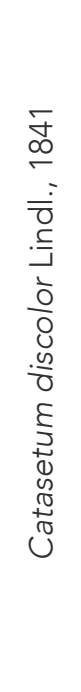 & 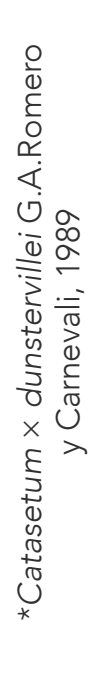 & 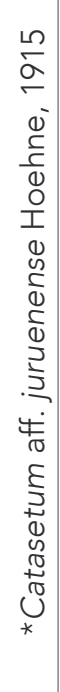 & 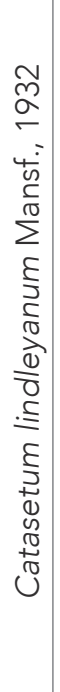 & 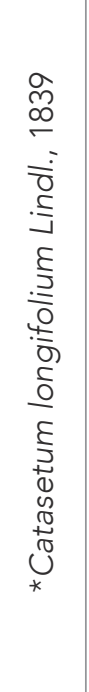 & 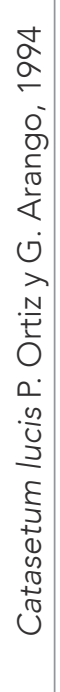 & 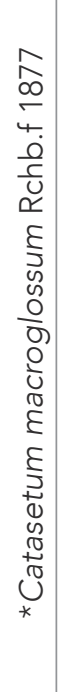 \\
\hline
\end{tabular}




\begin{tabular}{|c|c|c|c|c|c|c|c|c|c|c|c|c|c|c|c|c|c|c|c|c|c|}
\hline $\begin{array}{l}8 \\
\text { ధి } \\
\text { Dे }\end{array}$ & $\frac{8}{\stackrel{8}{1}}$ & $\begin{array}{l}\text { Oे } \\
\text { லे } \\
\stackrel{0}{0}\end{array}$ & 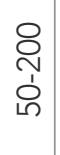 & $\begin{array}{l}8 \\
8 \\
\dot{1} \\
8\end{array}$ & 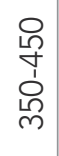 & $\begin{array}{l}8 \\
\stackrel{2}{1} \\
\grave{1} \\
8 \\
\infty\end{array}$ & $\begin{array}{l}8 \\
\frac{8}{2} \\
\frac{1}{8} \\
\frac{0}{1}\end{array}$ & $\begin{array}{l}\text { 㝵 } \\
\text { ○े } \\
\text { ڤn }\end{array}$ & $\begin{array}{l}8 \\
\frac{0}{c} \\
\text { in }\end{array}$ & 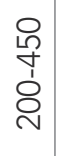 & $\begin{array}{l}8 \\
\text { } \\
\text { o } \\
\text { م }\end{array}$ & $\begin{array}{c}\stackrel{8}{\curvearrowright} \\
\stackrel{丶}{8} \\
\stackrel{\gamma}{2}\end{array}$ & 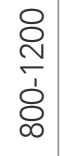 & $\begin{array}{l}8 \\
\frac{8}{8} \\
\frac{+}{+}\end{array}$ & 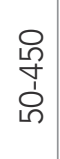 & 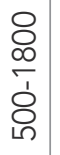 & ৪ & $\begin{array}{l}8 \\
\frac{8}{2} \\
\frac{1}{2} \\
\text { m }\end{array}$ & ৪্ & $\begin{array}{l}8 \\
0 \\
\text { ’े } \\
\text { ơ }\end{array}$ & $\begin{array}{l}8 \\
\text { f } \\
0\end{array}$ \\
\hline 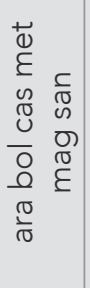 & 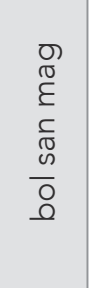 & $\sum_{\sigma}^{\sigma}$ & $\begin{array}{l}\tilde{E} \\
\frac{E}{\sigma}\end{array}$ & 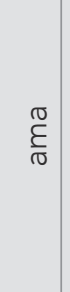 & $\underset{\pi}{\frac{\pi}{\sigma}}$ & $\begin{array}{l}\widetilde{ల} \\
\infty \\
\infty\end{array}$ & 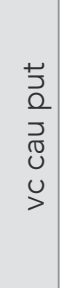 & $\begin{array}{l}\frac{u}{\lambda} \\
+ \\
\tilde{U} \\
\varepsilon\end{array}$ & 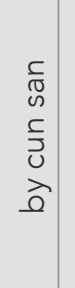 & $\begin{array}{l}\overrightarrow{0} \\
\tilde{E} \\
0 \\
\widetilde{U} \\
\tilde{\sigma} \\
\frac{\sigma}{\sigma}\end{array}$ & 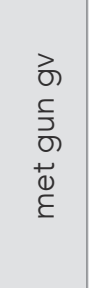 & $\begin{array}{l}\overrightarrow{\tilde{c}} \\
\omega \\
\tilde{\sigma} \\
\frac{\tilde{\sigma}}{\sigma}\end{array}$ & $\begin{array}{l}\vec{n} \\
\omega \\
\bar{\Omega}\end{array}$ & 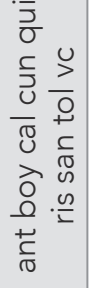 & $\stackrel{+}{E}$ & $\begin{array}{l}\stackrel{\overline{\mathbb{D}}}{\infty} \\
\infty\end{array}$ & $\begin{array}{l}\stackrel{5}{\mathbb{N}} \\
\stackrel{心}{\infty}\end{array}$ & $\begin{array}{l}\mathbb{N} \\
\tilde{D} \\
u \\
y\end{array}$ & 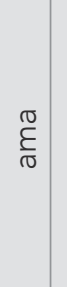 & $\bar{u}$ & $\begin{array}{l}\vec{r} \\
\omega \\
o \\
\frac{0}{u}\end{array}$ \\
\hline 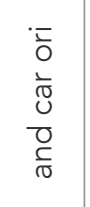 & $\begin{array}{l}\frac{\pi}{0} \\
\frac{0}{0} \\
\frac{0}{\sigma}\end{array}$ & $\frac{\sum_{\sigma}^{N}}{\pi}$ & 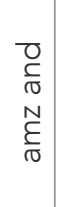 & $\sum_{\sigma}^{N}$ & $\begin{array}{l}\mathbb{N} \\
\frac{E}{\sigma} \\
\end{array}$ & 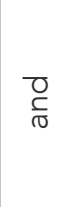 & 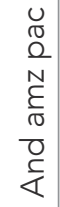 & $\bar{\partial}$ & $\frac{\bar{O}}{\frac{\tau}{\sigma}}$ & '̄ & 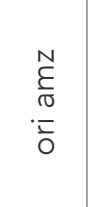 & $\begin{array}{l}\frac{N}{\sigma} \\
\frac{1}{\sigma} \\
\frac{\tilde{O}}{\sigma} \\
\frac{E}{\sigma}\end{array}$ & $\begin{array}{l}\bar{O} \\
\frac{\sigma}{\sigma} \\
0\end{array}$ & 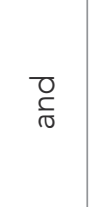 & $\overline{0}$ & $\begin{array}{l}\overline{0} \\
\frac{c}{\pi}\end{array}$ & $\begin{array}{l}\overline{0} \\
\frac{c}{\sigma}\end{array}$ & $\begin{array}{l}\bar{\sigma} \\
\frac{\Gamma}{\sigma}\end{array}$ & $\underset{\widetilde{\sigma}}{N}$ & $\begin{array}{l}\mathcal{N} \\
\frac{N}{\sigma}\end{array}$ & 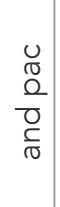 \\
\hline 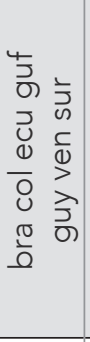 & 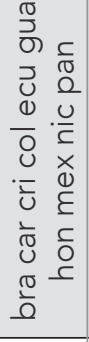 & $\frac{\stackrel{\complement}{\Phi}}{\stackrel{0}{\mathcal{O}}}$ & 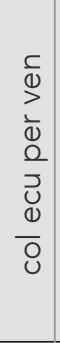 & 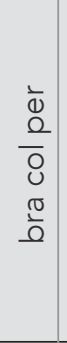 & $\begin{array}{l}\vec{z} \\
0 \\
\dot{0} \\
\stackrel{0}{0} \\
0 \\
0\end{array}$ & $\begin{array}{l}\frac{1}{0} \\
\frac{1}{0} \\
\stackrel{2}{0} \\
\\
\end{array}$ & $\begin{array}{l}\frac{c}{0} \\
\frac{2}{0} \\
0 \\
\frac{\pi}{0} \\
\end{array}$ & 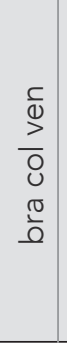 & 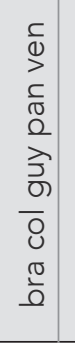 & $\overline{\mathrm{o}}$ & 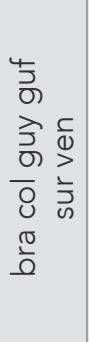 & 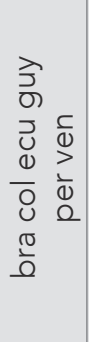 & $\frac{\bar{c}}{\stackrel{0}{0}}$ & $\frac{8}{\frac{0}{0}}$ & $\begin{array}{l}\bar{c} \\
\bar{D} \\
\frac{\sigma}{0} \\
\overline{0} \\
\overline{0}\end{array}$ & 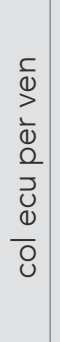 & $\overline{\mathrm{O}}$ & 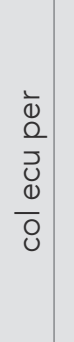 & $\begin{array}{l}\frac{\grave{d}}{\alpha} \\
\frac{0}{0}\end{array}$ & $\overline{\mathrm{o}}$ & 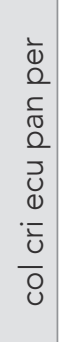 \\
\hline 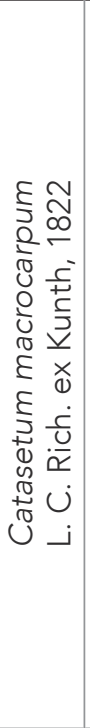 & 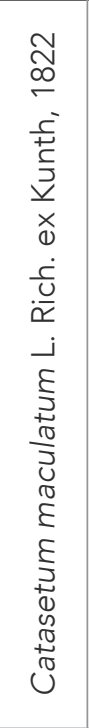 & 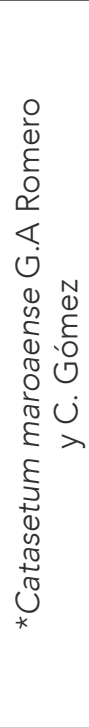 & 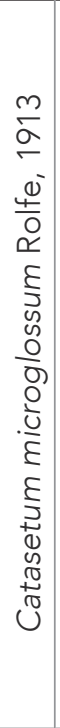 & 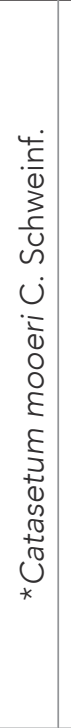 & 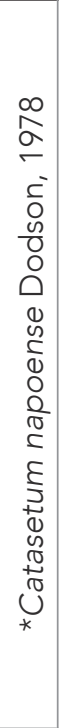 & 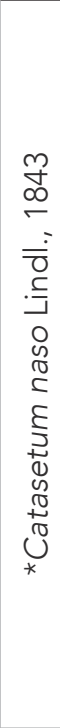 & 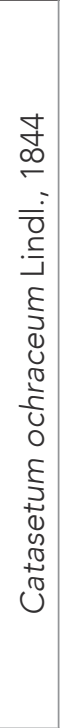 & 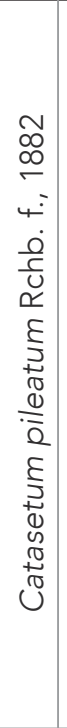 & 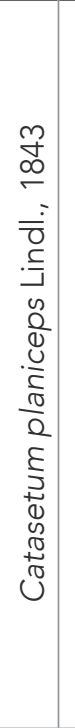 & 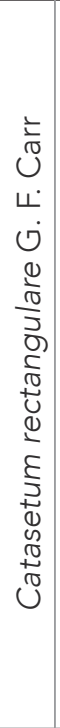 & 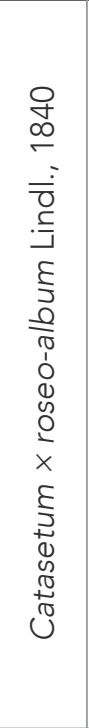 & 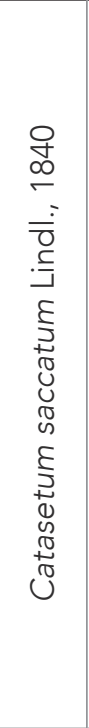 & 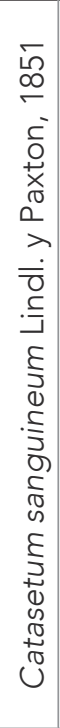 & 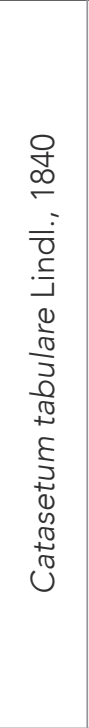 & 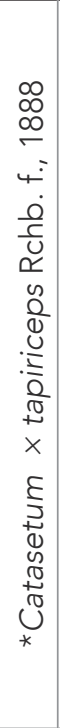 & 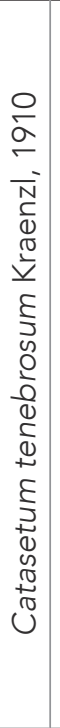 & 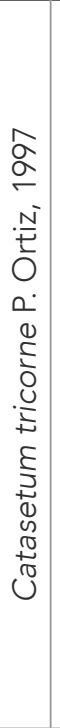 & 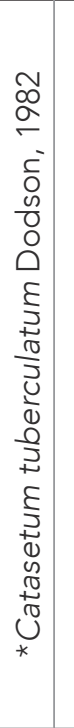 & 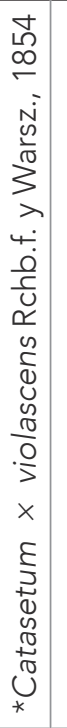 & 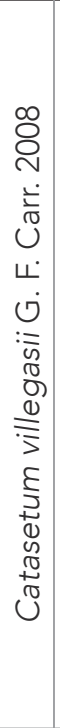 & 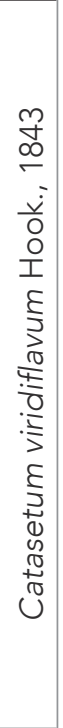 \\
\hline
\end{tabular}


Catasetum maculatum es una especie con distribución desde México hasta Brasil y en Colombia sólo se ha registrado para los departamentos de Bolívar, Magdalena y Santander, y encontrada a un rango altitudinal de 0 a 1.200 msnm, particularmente en la bioregión Andina y Caribe, en zonas de bosque seco (Tabla 1). Igualmente, López (2011) registra que en Santander crece en el mismo bioma con relación a la orografía andina y valle interandino del magdalena medio. En Ortiz y Uribe (2007), la especie que se determina como $C$. integerrinum es confundida con C. maculatum (Fig. 2) que es muy afín. Sin embargo, la principal diferencia está en que el borde del labelo se dobla hacia dentro, particularmente inclinado hacia la columna, opuesto a lo conocido en C. maculatum (Holst, 1999).

\section{Distribución}

La distribución de género Catasetum va desde México hasta Brasil y Norte de Argentina, concentrado la mayor diversidad se sus especies en Brasil, centro de diversidad del grupo (Romero, 2009). En

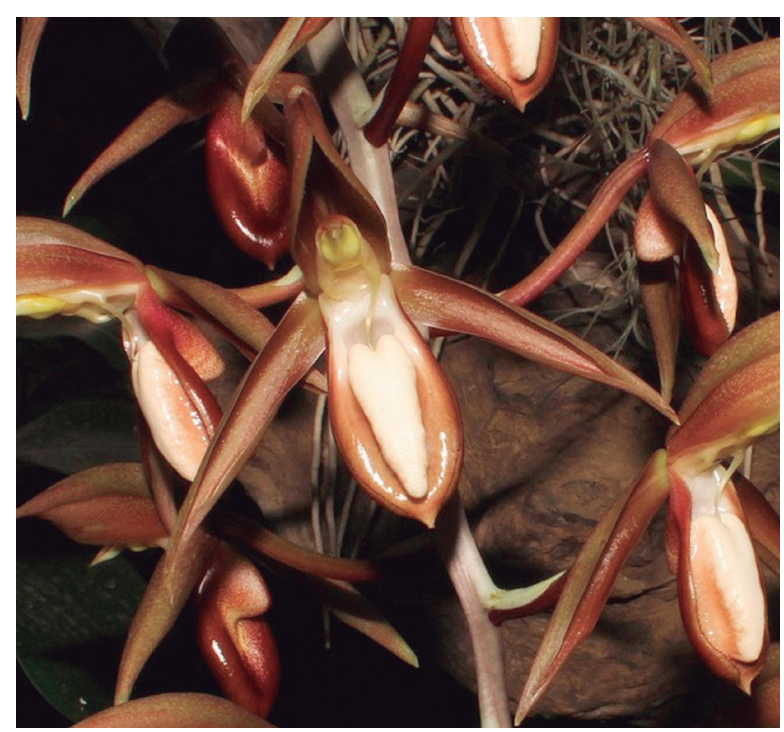

Figura 1. Flor masculina de Catasetum tabulare. Foto: Miguel Bonilla y Carolina Aguirre.

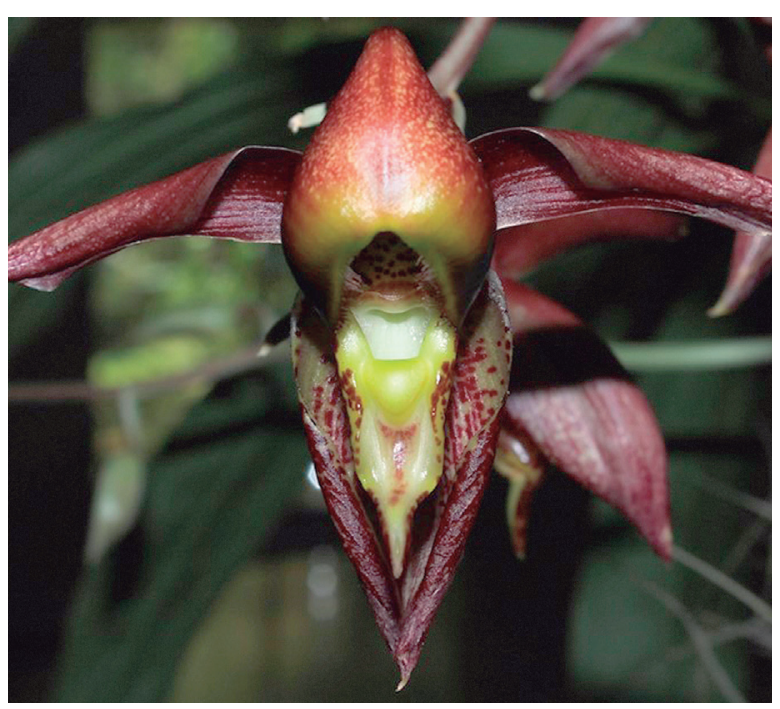

Figura 2. Flor masculina de Catasetum maculatum. Foto: Miguel Bonilla y Carolina Aguirre.

Colombia, Catasetum es un grupo que se encuentra en la mayoría de los departamentos, sin embargo, la bioregión de la Amazonía presenta escasas colectas que incluyen a Vaupés sin ninguna, al igual que el Norte de Santander en los Andes, y aún más representativo en el Atlántico con vacíos de colecta en Atlántico, Córdoba, Sucre, Guajira y Cesar (Fig. 3).

Estudios preliminares realizados en taxonomía y biogeografía de Catasetum por Bonilla et al. (2013a), determinan que el grupo principalmente se concentra el mayor número de especies en bosques secos de la bioregión andina, valles interandinos y asociadas al piedemonte, principalmente, relacionados con la Cordillera Oriental y Central, entre los 400 a 1200 msnm. A nivel Neotropical, las relaciones ecogeográficas definen que es un grupo que va desde el nivel del mar hasta los 2200 msnm, especialmente $C$. discolor que crece en los Tepeuyes de Guyana Francesa (Romero, 2009).

\section{Comentarios de cultivo}

En Colombia algunas especies se utilizan en cultivo como C. callosum (Cebolleta), C. collare (Enanos) 
que son confundidas con C. gnomus que no posee registros en el país, C. macrocarpum (Cebolleta), C. pileatum (Cebolleta de San Martín) y C. tabulare (Dios te dé) de uso ornamental (Carvajal, 2004). Especies ampliamente cultivadas o abundantes en sus nichos y que son recogidas como plantas ornamentales son $C$. $\times$ roseo-album (angelito rosado), C. sanguineum (flor rinoceronte), C. rectangulare (bicho), C. cristatum (peluda), C. lucis (flor con falda), C. bicolor (flor del cruxificado), C. maculatum (niño manchado), C. planiceps (soldadito) y C. viridiflavum (casco). En las últimas décadas se ha incrementado su interés y valor hortícola, particularmente, por su capacidad de generar híbridos tanto naturales como artificiales (Viera, 1990).
No obstante, para algunas especies se confunde su identidad taxonómica o no se conoce su distribución.

\section{Comentarios taxonómicos de especies poco conocidas}

En Galería de Orquídeas de Ortiz y Uribe (2007), se confunde C. callosum con C. rectangulare (Fig. $4)$, por tal motivo, es importante tener referencia de los caracteres diagnósticos para la determinación del complejo de especies que componen el grupo Callosum. Este error se repite en el trabajo de Ortiz y Uribe (2014) donde, a partir de registros fotográficos, se confunde la identidad de las dos especies (C. rectangulare y C. callosum). La planta con la que

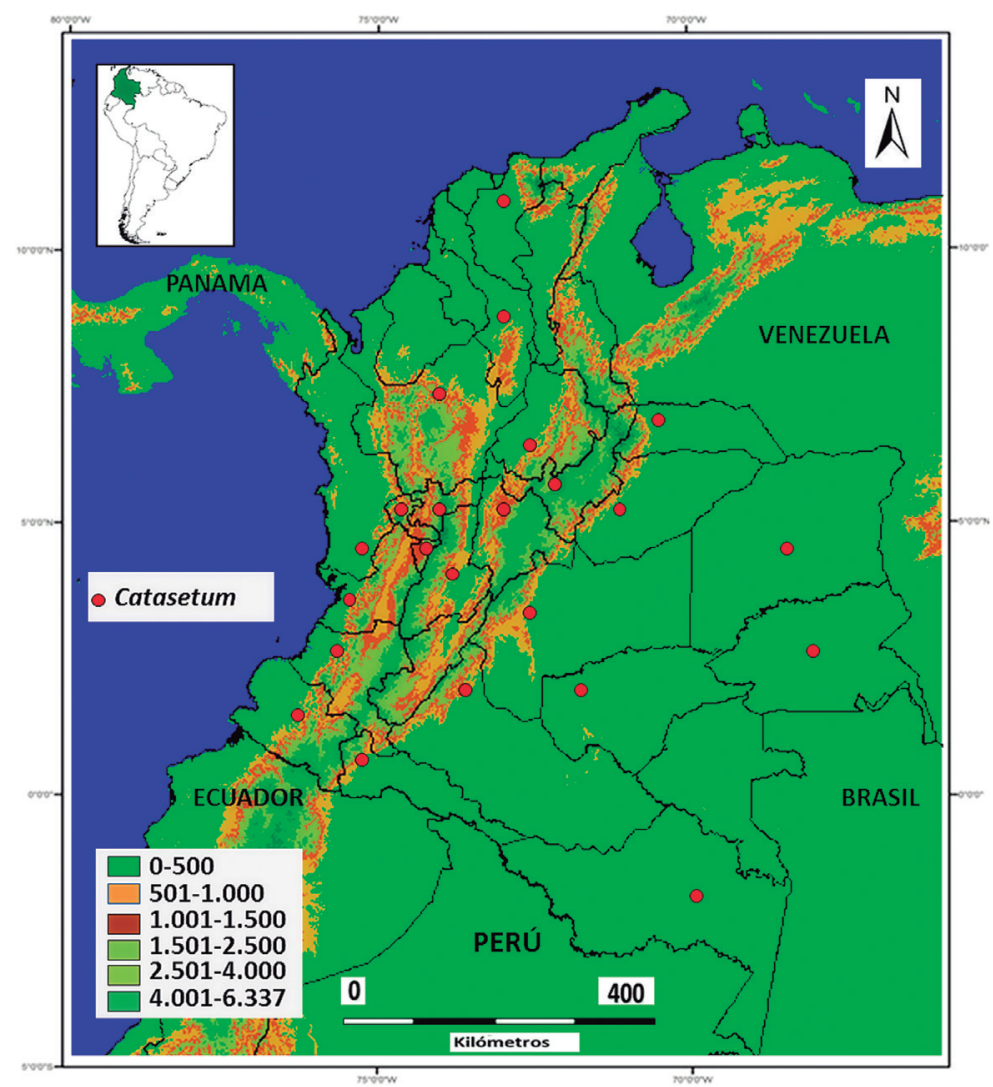

Figura 3. Distribución de Catasetum por departamentos en Colombia. 
determinaron el tipo de C. rectangulare posiblemente es obtenida en la Orinoquía, departamento de Arauca (Carr, 2006). Estudio de diversidad y distribución para esta especie, realizados por Bonilla et al. (2014a), registraron su presencia desde el Meta hasta Arauca, con un rango altitudinal de 182 a 582 msnm, asociada al piedemonte bajo andino, piedemonte y altillanura. Su distribución es exclusiva de Colombia por lo que se considera endémica de la Orinoquía. Los caracteres diagnósticos para su determinación son: difiere de C. callosum Lindl. por el labelo angosto y rectangular y los sépalos laterales falcados (Carr, 2006).

(A)

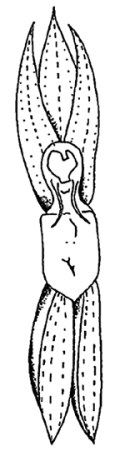

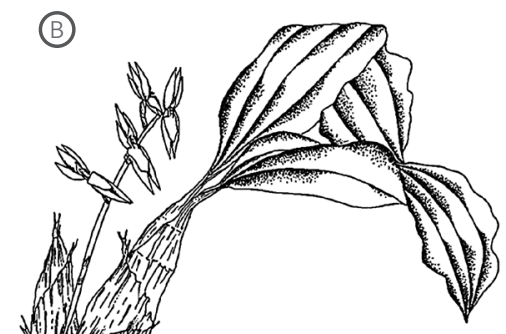

Figura 4. Catasetum rectangulare. a) flor unisexual masculina, b) planta con inflorescencia. Ilustración: Miguel Bonilla y Diego Yepes.

Catasetum villegasii (Fig. 5) solamente ha sido reportado para el departamento de Caquetá, al suroeste de Florencia, a una altitud entre 400 a 600 msnm, con un período de floración de agosto a septiembre, bajo cultivo (Carr, 2008). Esta especie es endémica de Colombia, conocida sólo de la localidad tipo y su caracteres diagnósticos hacen relación al labelo con uñas, no lobulados, elíptico-ovado y ampliamente triangular en el ápice, agudo, margen entero con forma de platillo (Carr, 2008).

Catasetum aff. $\times$ apolloi es una especie reportada para Brasil, sin embargo en Colombia posiblemente se encuentra en Arauca (Fig. 6), de acuerdo

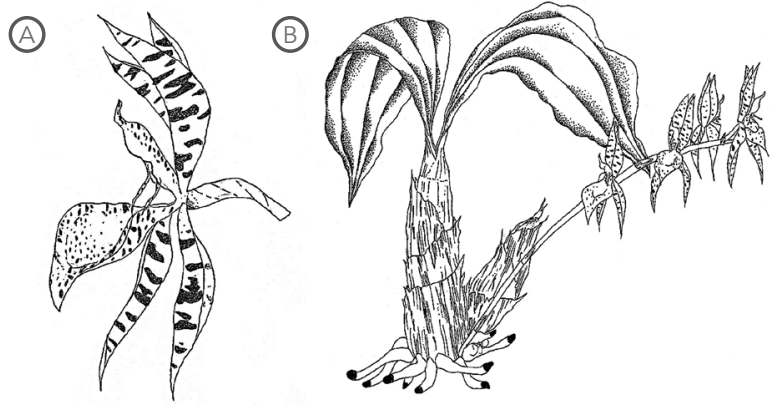

Figura 5. Catasetum villegasii. a) flor unisexual masculina, b) Planta con inflorescencia. Ilustración: Diego Yepes y Miguel Bonilla.

con la planta colectada por Diana García y tratada en Bonilla et al. (2013a). Además, se encuentra en el rango altitudinal reportado para la especie, de 300-400 msnm (Benelli y Grade, 2008). Después de ensayos de cruzamientos interespecíficos de C. schmidtianum F.E.L \& K. G. L y C. longifolium Lindley se determinó que es un híbrido entre estos parentales, pasando C. apolloi a C. $\times$ apolloi, por ser un híbrido natural (Benelli y Grade, 2012). En la región de la Orinoquía se ha reportado $C$. longifolium e hibrido natural de esta especie como C. $\times$ roseo-album, sin embargo, no se conocen reportes de C. schmidtianum (Bonilla et al., 2013a). Los caracteres diagnósticos son pétalos abovados, ápice obtuso, reflexo, ligeramente

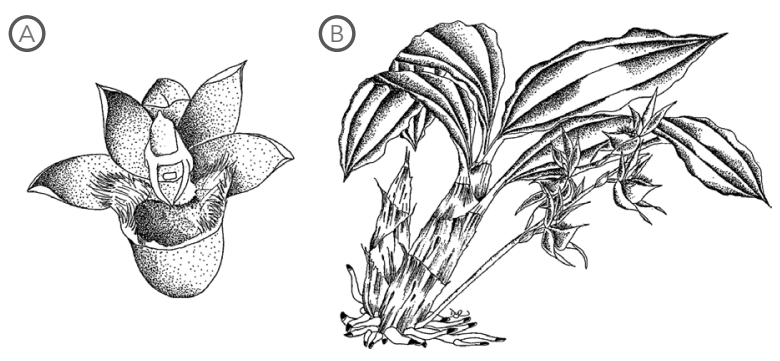

Figura 6. Catasetum aff. $\times$ apolloi. a) flor masculina, b) planta con inflorescencia. Ilustración: Diego Yepes y Miguel Bonilla. 
cóncavo (29 × $16 \mathrm{~mm}$ ); sépalo dorsal oval ligeramente cóncavo (24 mm de longitud); sépalos laterales triangulares (27 mm de largo); labelo ostium orbicular, saco (15 mm de profundidad y $12 \mathrm{~mm}$ de ancho), márgenes fimbriados hasta la base, lóbulo medio lingüiformey ápice agudo (Benelli y Grade, 2008).

Catasetum $\times$ dunstervillei (Fig. 7) es un híbrido natural entre $C$. discolor y $C$. pileatum, reportado en Guyana y Venezuela en un rango altitudinal de 50-200 msnm (Carnevali et al. 2003). En Colombia ha

(A)

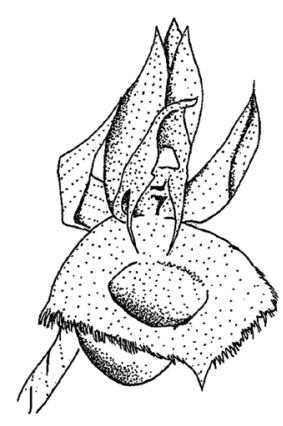

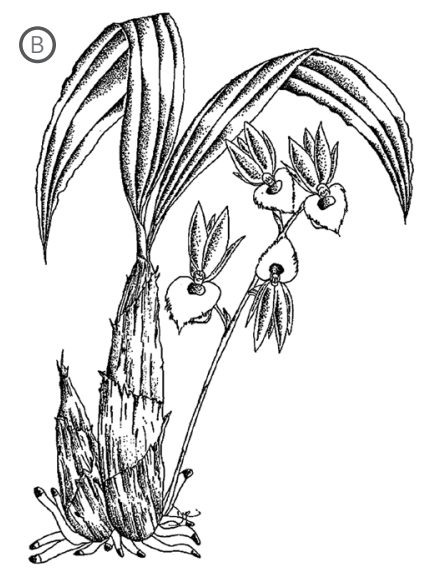

Figura 7. Catasetum $\times$ dunstervillei. a) flor masculina modificada de Carnevali et al. (2003), b) planta con inflorescencia. Ilustración: Diego Yepes y Miguel Bonilla.

sido encontrada en el departamento del Meta (Villavicencio) a 450-500 msnm con las poblaciones parentales y mantenida in vivo (Jiménez, 2011; Bonilla et al. 2013a), además de poseer presumibles reportes en Puerto López (Villegas, 2002). En cuanto los caracteres diagnósticos: sépalo dorsal cóncavo, lanceolado, acuminado, erecto (23-25 × 9-10 mm); sépalos laterales cóncavos, obovado, ligeramente oblicuo, apiculado, recurvo (22-25 x 9-10); pétalos convexo, abovado, ligeramente oblicuo, apiculado (25-27 x 11-13); labelo sacciforme (sacado), profundidad del saco 15-18 mm, dos hendiduras poco profundas junto al ápice, ligeramente trilobulado y con un callo en forma de " $\mathrm{V}$ " cercano al margen ápical interno, margen reflexo finamente denticulado o fimbriado (Romero y Carnevali, 1989b).

Catasetum $\times$ tapiriceps (Fig. 8) es un hibrido natural entre C. macrocarpum y Catasetum pileatum Rchb. f., se ha reportado en Brasil, Colombia y Venezuela a 50-100 msnm (Carnevali et al., 2003). En Colombia se ha registrado en el Meta y Casanare entre 300 a 450 msnm (Jiménez, 2011; Bonilla et al. 2013b). En el trabajo de Ortiz y Uribe (2007), se encuentra un dibujo sin localidad, y en Ortiz y Uribe (2014) se confunde con algunas fotos de C. macrocarpum y se determina con el sinónimo de C. x splendens Cogn. Los caracteres diagnósticos son flores blancas, verdes, verdosas o amarillas, frecuentemente con manchas cafés; labelo con prominencia, con callo transversal cercano al ápice, que puede ser entero o 3 lobulado, el lóbulo medio plano o en canal, truncado, obtuso o retuso colgado (Carnevali et al. 2003).

Ortiz y Uribe (2007) en su trabajo de galería de fotos incluye como especie no identificada a Catasetum mooeri (Fig. 9 a, b), sin embargo, el sitio de colecta es incierto, con altas probabilidades de que haga parte de la Amazonía, asociada a la frontera con Brasil donde ha sido reportada entre 200 a 900 msnm. Sin embargo, también existe un Catasetum
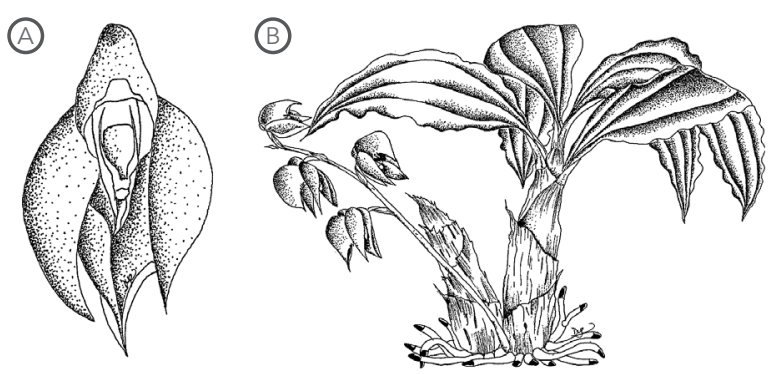

Figura 8. Catasetum $\times$ tapiriceps. a) flor masculina, b) planta con inflorescencia. Ilustración: Diego Yepes y Miguel Bonilla. 
sp. que fue registrada por Viera (1990) de planta en cultivo. En cuanto a los caracteres diagnósticos: sépalo dorsal elíptico, cóncavo, agudo o acuminado (3,5 x 1,4 cm); sépalo lateral, lanceolado-elíptico, acuminado, ligeramente oblicuo $(3,6 \times 1,6 \mathrm{~cm})$; pétalos ovado-elíptico, acuminado $(3,4 \times 1,6 \mathrm{~cm})$; labelo simple, ancho y ovado alrededor, curvo a los lados, subobtuso, callo en la base, ancho en la inferior $(2,5 \times 2,6 \mathrm{~cm})$, margen minuciosamente denticulado (Schweinfurth, 1970).

Catasetum dejeaniorum (Fig. 9c) ha sido colectada en la Guyana Francesa a un rango altitudinal de 200-300 msnm (Chiron, 2007). Ortiz y Uribe (2007), incluye esta especie en su galería de fotos, sin embargo, la determina como C. planiceps, especie muy cercana. Sumado, Ortiz y Uribe (2014) identifican esta misma foto como Catasetum decipiens Rchb.f., especie que presenta notables diferencias asociadas al margen del labelo revoluto y fimbriado. Aunque la distribución de la especie no concuerda, se considera que análisis genéticos de C. planiceps y $C$. dejeaniorum determinarán diferencias interespecíficas, pues a nivel morfológico se consideran especies estrechamente relacionadas.

En adenda a las orquídeas de Santander (López, com. pers., 2015) se incluye Catasetum tenebrosum (Fig. 10) encontrada en una palma, a $500 \mathrm{msnm}$ en Puerto Wilches. Además, esta especie ha sido encontrada en Ecuador, Perú y extremo oeste de Brasil, registrada en hábitat natural de 800 a 1500 msnm (Holst, 1997). Los caracteres diagnósticos son flores de color chocolate a un purpura parduzco y labelo verdoso amarillento característico de esta especie. Sépalo dorsal elíptico-oblongo, agudo o cortamente acuminado $(3-3,5 \times 1,4 \mathrm{~cm})$; sépalos laterales, similares ligeramente oblicuos; pétalos muy similar al sépalo dorsal; labelo delgado, triangularovado, entero, obtuso, base ligeramente cordada, suculenta y márgenes crenulados a dentados, disco ligeramente convexo, con un centro conspicuo similar a una perilla en la base y ápice obtuso (Schweinfurth, 1960).
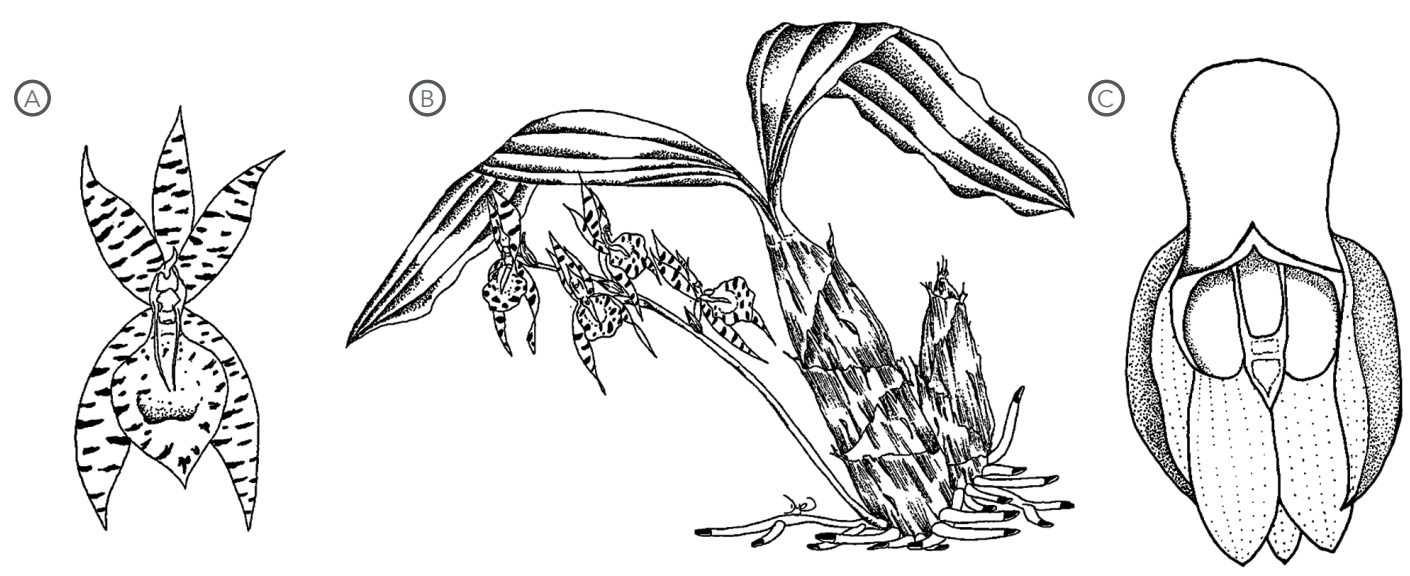

Figura 9. Catasetum moeeri (a y b) y C. dejenearium (c). Ilustración: Diego Yepes y Miguel Bonilla. 

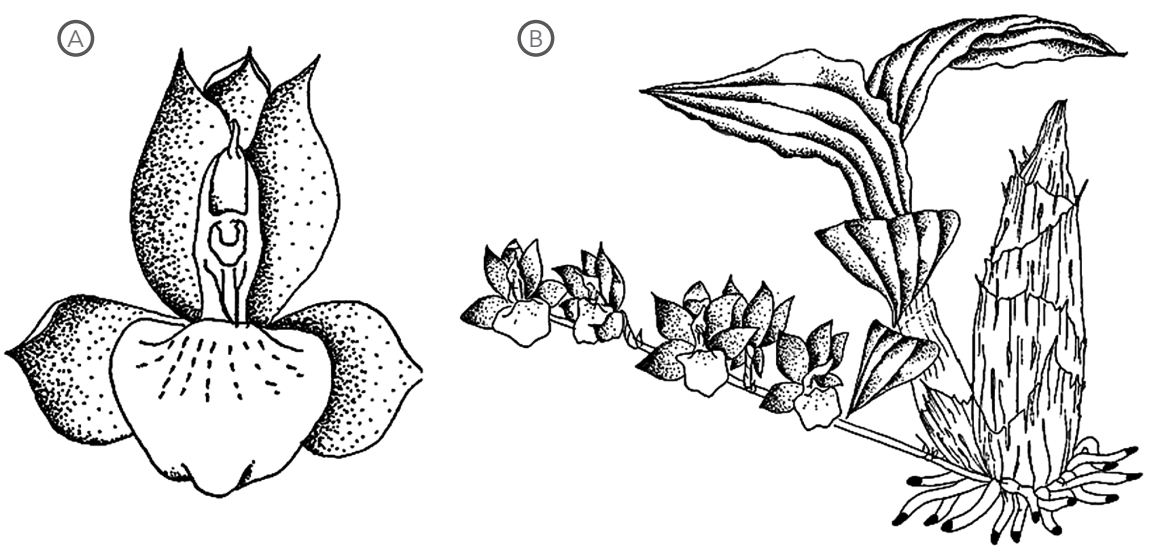

Figura 10. Catasetum tenebrosum. a) flor masculina, b) planta con inflorescencia. llustración: Diego Yepes y Miguel Bonilla.

\section{CONCLUSIONES}

Los resultados permiten deducir que los Catasetum de Colombia son un grupo poco estudiado y que probablemente la cifra de especies para el país siga creciendo. De tal manera, futuros programas de colecta deben enfocarse en los GAP's de la Amazonía y Atlántico, principalmente, dada su correlación con la Amazonía brasileña, centro de diversidad del grupo. Esto permitirá profundizar el estudio del género y así conocer su distribución y parámetros ecológicos con enfoque al establecimiento de programas de manejo y conservación in situ y ex situ.

\section{AGRADECIMIENTOS}

Los autores agradecen a Colciencias y a la Universidad Nacional de Colombia Sede Palmira por la financiación y apoyo del proyecto "Relaciones ecológicas y evolutivas entre orquídeas (Orchidaceae: Catasetinae y Stanhopeinae) y sus polinizadores, las abejas euglosinas (Apidae: Euglossini), en la región amazónica de Putumayo y pacífica de Nariño, Colombia" código 112065945149 número de convocatoria 569. También a Holguer López Toscano y Adarilda Benelli por compartir sus conocimientos y motivar el presente trabajo. A Diana García y cultivadores de orquídeas de Colombia por permitir acceder a sus colecciones y brindar la información correspondiente de sus plantas. 


\section{REFERENCIAS}

- $\quad$ Benelli A y Grade A. 2008. A new species of Catasetum (Orchidaceae) for the state of Mate Grosso, Brasil. Edinb J Bot 2008; 65 (1): 27-34.

- Benelli A, y Grade A. 2012.Catasetum apolloi Benelli \& Grade (Orchidaceae): correcction taxonomique. Richardiana. 12(4): 153-157.

- Bonilla M, Yepes D y Otero J. 2014a. Diversidad y distribución de Catasetum rectangulare G.F. Carr (Orchidaceae: Catasetinae). Orquideología. 31 (1): 15-25.

- Bonilla M, Gallego E y Aguirre C. 2014b. Notas sobre Galeandra beyrichii Rchb. f. (Orchidaceae: Catasetinae) para Colombia. Orquideología. 31 (1): 33-44.

- Bonilla M, Gallego E y Otero J. 2013a. Biogeografía y Taxonomía de Catasetum. Memorias VII Congreso Colombiano de Botánica. 252pp.

- Bonilla M, Otero J. y Durán E. 2013b. Lista preliminar de Catasetinae (Orchidaceae) en Colombia. Memorias VII Congreso Colombiano de Botánica. 544p.

- Camargo C y Delgado A. 2006. Flora Orchidaceae de la mesa de los santos (Piedecuesta - Santander). Trabajo de grado Universidad Industrial de Santander. 306 pp.

- Carvajal E. 2004. Plantas útiles y de uso común en Colombia. Universidad Francisco de Paula Santander, Facultad de Ciencias Agrarias y del Ambiente, San José de Cucutá. 128 pp. Disponible: http://www.cucutanuestra.com/ temas/flora_cucuta/nombre_comun_y_cientifico/COMPLEMENTOS\%20DE\%20CATEGORIAS\%20TAXONOMICAS.pdf

- Chase M, Cameron K, Freudenstein J, Pridgeon A, Salazar G, Van Den Berg C y Schuiteman A.
2015. An updated classification of Orchidaceae. Botanical Journal of the Linnean Society. 177:151-174.

- Carnevali G, Ramírez I, Romero A, Vargas C y Foldats E. (2003).Orchidaceae. In: Flora of the Venezuelan Guayana. P. E. Berry et al. (eds.), Volumen 7. Missouri Botanical Garden, St. Louis. 200-619pp.

- Carr G. 2006. Catasetum rectangulare. Orchids Review.114 : 32-33.

- Carr G. 2008. A new Catasetum from Colombia. Orchids Review. 116: 226-228.

- Viera L. 1990. Catasetum. Escobar, R (Ed). Orquídeas nativas de Colombia (A-D). Sociedad Colombiana de Orquideología. 139pp.

- $\quad$ Freudenstein J, y Mark W. 2015. Phylogenetic relationships in Epidendroideae (Orchidaceae), one of the great flowering plant radiations: progressive specialization and diversification. Annals of Botany. 1-17.

- Gerlach G.2013. La pesadilla de Lindley- La biología sexual de Catasetum y Cycnoches. Lankesteriana. 3 (1-2): 39-46.

- Giraldo G, y Betancur, J. 2012 Guía de campo de las orquídeas de santa maría (Boyacá, Colombia). Serie de guía de campo del instituto de ciencias naturales, universidad nacional de Colombia.187pp.

- Holst A. 1999. The world of Catasetums. Timber Press, Inc. 271pp.

- Jiménez C. 2011. Orquídeas del Meta. 179pp.

- López H. 2011. Orquídeas nativas de Santander. Gobernación de Santander. 319 p.

- Martínez S, Bonilla M y López H. 2015. Lista de Orchidaceae de Santander y comentarios 
sobre sus especies endémicas. Revista de la Facultad de Ciencias Básicas, 11 (2): 54-111.

- Mejía E. (2009). Orquídeas del Quindío, Asociación Quindiana de Orquideología-CRQ, pp. 216.

- Misas G. 2005.Orquídeas de la Serranía del Baudo. Corporación Capitalina de Orquideología. p.792.

- $\quad$ Ortiz P, y Uribe C. 2007. Galería de Orquídeas de Colombia. CD Edition, Bogotá-Colombia, Asociación Bogotana Orquideológica.

- Ortiz P, y Uribe C. 2014. Orquídeas, tesoro de Colombia (A-D). Editorial Da Vinci Publicidad y Medios \& cía. S. En. C. 397 pp.

- $\quad$ Ortiz P. 2015. Catasetum. En Bernal R., Grasdstein S. y Celis M. Catálogo de plantas y líquenes de Colombia. Instituto de Ciencias Naturales de Colombia, Bogotá. http://catalogoplantascolombia.unal.edu.co

- Pérez O, Sánchez E y Valdivieso O. 2009. Inventario orquideológico de la Reserva Bosque de Yotoco, Valle del Cauca. Acta Agronómica. 58 (3): 189-196.

- Reina G, Ospina N, Castaño A, Soriano l y Otero J. 2010. Catálogo de las orquídeas del valle geográfico del río Cauca y su piedemonte bajo. Sur-Occidente Colombiano. Cespedesia. 32 (90-91): 7-22.

- Romero G, y Carnevali G. 1989a. Novelties in the Orchid Flora of Southern Venezuela. Ann. Mo Bot Gard 1989; 76: 454-461.

- Romero G, y Carnevali G. 1989b. A new combination for the Orchid Flora of Southern Venezuela: Catasetum tapiriceps Reichb. f. (pro. sp.). Lindleyana. 4: 127-134.

- Romero G, y Jenny R. 1993. Contributions toward a monograph of Catasetum
(Catasetinae, Orchidaceae) I: A checklist of species, varieties, and natural hybrids. Harvard Papers. 4: 59-84.

- Romero G. 2009: Subtribes Catasetinae. In: Genera Orchidacearum, Vol. 5 Epidendroidae (Part two). Pridgeon, Cribb, Chase, Rasmussen, Eds., Oxford University Press, Oxford, New York. 11-12.

- Romero G. 2012. Las flores unisexuales y dimórficas de Catasetum Rich. (Orchidaceae). Herbario CICY. 4: 32-36.

- Sarmiento J. 2007. La familia Orchidaceae en Colombia, Actualidades biológicas. 29 (Supl. 1), 21-84.

- Schweinfurth C. 1960. Fieldiana Botany: First supplement to the Orchids of Perú. Field Museum of Natural History. 30 (3). 591 pp.

- Schweinfurth C. 1970. Fieldiana Botany: First supplement to the Orchids of Perú. Field Museum of Natural History. 30. p. 50-51.

- Villegas F. 2002. Catasetum pileatum x Catasetum roseo-album un híbrido natural de los Ilanos orientales. Orquideología. 22:165-168.

- Whitten M, Neubig K y Williams N. 2014. Generic and subtribal relationships in neotropical Cymbidieae (Orchidaceae) based on matK/ ycf1 platid data. Lankesteriana.13 (3): 375-392.

- Zotz G. 2013. The systematic distribution of vascular epiphytes - a critical update. Bot J Linn Soc. 171: 453-481. 